\title{
HA NDBUCH
}

\section{DER \\ M INER A LOGIE. \\ VoN}

DR. CARL HINTZE,

o. ö. PROFESSOR AN DER UNIVERSITÄT BRESLAU.

ZWEITER BAND.

SILICATE UND TITANATE.

MIT 632 ABBILDUNǴEN IM TEXT.

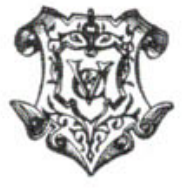

LEIPZIG,

VERLAG VON VEIT \& COMP.

1897. 
Druck von Metzger \& Witig in Leipzig. 